\title{
Feasibility of polarized all-sky imaging for aerosol characterization
}

\section{A. Kreuter and M. Blumthaler}

Division for Biomedical Physics, Department of Physiology and Medical Physics, Innsbruck Medical University, Muellerstr. 44, 6020 Innsbruck, Austria

Correspondence to: A. Kreuter (axel.kreuter@i-med.ac.at)

Received: 15 November 2012 - Published in Atmos. Meas. Tech. Discuss.: 14 December 2012

Revised: 13 June 2013 - Accepted: 24 June 2013 - Published: 31 July 2013

\begin{abstract}
In this study, we investigate the method of polarized all-sky imaging with respect to aerosol characterization. As a technical frame work for image processing and analysis, we propose Zernike polynomials to decompose the relative Stokes parameter distributions. This defines a suitable and efficient feature vector which is also appealing because it is independent of calibration, circumvents overexposure problems and is robust against pixel noise. We model the polarized radiances of realistic aerosol scenarios and construct the feature vector space of the key aerosol types in terms of the first two principal components describing the maximal variances. We show that, using this representation, aerosol types can be clearly distinguished with respect to fine and coarse mode dominated size distribution and index of refraction. We further investigate the individual influences of varying aerosol properties and solar zenith angle. This suggests that polarized all-sky imaging may improve aerosol characterization in combination with sky scanning radiometers of the existing Aerosol Robotic Network (AERONET) especially at low aerosol optical depths and low solar zenith angles.
\end{abstract}

\section{Introduction}

Aerosols in the atmosphere absorb and scatter solar radiation and hence modify the earth's radiation budget. In addition to this direct effect, several indirect effects involving cloud formation add to the aerosols' complex impact on climate that are relatively poorly understood (Forster et al., 2007). One important step towards a better representation of aerosols in future climate models is to improve global aerosol characterization from the observational side.

Aerosol distribution and properties may vary strongly on both temporal and spatial scales, so satellite observations of aerosols would be ideally suited (Kaufman et al., 2002). However, satellite retrievals are of limited quality over surfaces with high albedo such as deserts and snow cover. Ground based remote sensing remains a key method to determine aerosol properties with high accuracy and is indispensable for satellite validation.

The physical observable in remote sensing is the sky radiance, which is measured at different viewing angles (angular distribution), in different wavelength bands and includes the polarization state. The radiance distribution is commonly measured with sky scanning radiometers with a small fieldof-view (FOV) input optics pointing at discrete viewing angles. These measurements constitute the input data for retrieval schemes for aerosol optical properties, such as the one used for the Aerosol Robotic Network (AERONET) (Holben et al., 1998; Dubovik and King, 2000).

An alternative method of measuring the sky radiance is all-sky imaging, i.e. projecting the polarized sky hemisphere onto a camera chip with a fisheye objective (see e.g. North and Duggin, 1997; Liu and Voss, 1997; Pust and Shaw, 2008; Cazorla et al., 2009; Kreuter et al., 2009). This method will be the subject of this study and we want to explore its feasibility for aerosol characterization. Compared to standard AERONET (sky scanning) measurements, all-sky imaging has advantages and disadvantages.

All-sky imagers capture the whole sky radiance distribution in multiple wavelength bands in one exposure. This is a clear advantage in terms of data acquisition speed of all observable viewing angles and wavelengths. However, simple sky imagers using photo camera sensors have three broadband wavelength bands to imitate the physiological color response, while sky scanners measure in selected narrow wavelength bands. In principal, narrow band response in all-sky 
imagers could be achieved by filter wheels or special on-chip filters.

Sky scanning instruments typically measure only two specific sections in the sky: the principal plane (PP), which is the vertical plane containing the sun and the zenith, and the so-called almucantar, which is a scan of the azimuth angle at a constant zenith angle, equal to the solar zenith angle (SZA). With respect to aerosol information, the almucantar becomes impractical for low SZA because of the decreasing amount of scattering angles. This problem may be mitigated when capturing all hemispheric viewing angles. The information of all viewing angles also implies the advantage of easier identification of cloud contamination. This is relevant for the retrieval of aerosol properties since the basic retrieval assumption is clear sky. With sky scanning instruments, the symmetry of the almucantar can be used to deduce clear sky conditions (Holben et al., 2013).

Sky scanners have a small FOV of $\sim 1.2^{\circ}$ and can thus measure the radiance also close to the sun. This is an advantage, as the so-called aureole contains the forward scattering angles and is particularly sensitive to coarse particle scattering. All-sky imagers with modern CCD sensors with $\sim 10^{4}$ pixels have a FOV $<1^{\circ}$. Nonetheless, the aureole cannot be resolved satisfactorily because of lens flare and overexposure. The difference of direct sun and sky radiance is beyond the dynamic range of the sensors.

Finally, typical photo camera sensors are not optimized for absolute radiometric stability and calibration is elaborate. Here, we will circumvent the calibration problem and, instead of the absolute radiance, we will focus on the polarization state of the radiance which can be expressed in relative units. Since aerosol scattering alters not only the sky radiance but also its polarization, polarization adds independent measurement degrees of freedom with respect to aerosols (e.g. Vermeulen et al., 2000; Boesche et al., 2006; Li et al., 2009).

Comparing these two complimentary methods, it is interesting to ask how all-sky imagers, despite its limitations, can be used for aerosol characterization. So in the following, we start out by developing a suitable image analysis method. Then we investigate the sensitivity of the proposed feature vector with respect to different aerosol properties. We consider the optical properties of key aerosol types observed in worldwide locations and model the corresponding polarized all-sky distributions. Then we show within a suitable representation, how these different types are distinguishable and investigate the effect of varying aerosol optical properties and SZA. Finally, we discuss uncertainties and information content and suggest the feasibility of this method to improve aerosol characterization.

\section{Method}

\subsection{The radiative transfer model}

The (linearly) polarized sky radiance is described by the three Stokes parameters $I, Q$ and $U$, defined for operational purposes as (e.g. Born and Wolf, 1999)

$$
\left(\begin{array}{c}
I \\
Q \\
U
\end{array}\right)=\left(\begin{array}{c}
I_{0}+I_{90} \\
I_{0}-I_{90} \\
I_{45}-I_{135}
\end{array}\right),
$$

where $I_{x}$ are the radiances measured at four polarizer orientation angles $x$, with respect to an arbitrary reference angle. So, in polarized all-sky imaging four images at these different polarizer orientations are recorded. Applying the definition in equation 1 at each pixel produces the Stokes parameter distributions, which we will simply call Stokes maps. The method is presented in more detail in Kreuter et al. (2010).

For the purpose of this study, we simulate Stokes maps for different aerosol scenarios using model calculations. The model we use is the Monte Carlo radiative transfer model MYSTIC which is planned to be publicly available in a future release of the libRadtran Package (Mayer and Kylling, 2005). The MYSTIC solver has been extensively validated (Kokhanovsky et al., 2010) and is described by (Mayer, 2009; Emde et al., 2010). Here we use a simplified 1-D version of MYSTIC, with the restriction of a homogeneous and flat surface, with ground albedo (Lambertian reflectance) set to 0.2 throughout this study.

We consider three wavelengths, 650, 550 and $450 \mathrm{~nm}$, roughly corresponding to the center wavelengths of the red $(\mathrm{R})$, green $(\mathrm{G})$ and blue (B) channels of typical RGB sensors. For simplicity here, we ignore the bandwidth associated with real RGB sensors since, in principal, narrow band filters can be used.

All simulations are performed with $3 \times 10^{4}$ photons resulting in a numerical noise of less than $1 \%$ relative standard deviation in the modeled radiances. The viewing angle grid is set up in Cartesian coordinates (rather than radial) to produce a constant solid angle for each pixel. Each map consists of $25 \times 25$ pixels, resulting in 441 viewing directions, with a FOV of $7.5^{\circ}$. This resolution allows for a fast computation of the Stokes maps and is sufficient for our study. In reality, the resolution of modern sensors may exceed $1000 \times 1000$ pixels, resulting in a FOV of $<1^{\circ}$.

\subsection{The aerosol model}

As model input the aerosol properties have to be specified, which may vary considerably depending on their origin. Various aerosol models for classification have been proposed in the past (e.g. Shettle and Fenn, 1979; Hess et al., 1998). Here, as representative realistic aerosols, we consider the four key aerosol types observed by AERONET in worldwide locations (Dubovik et al., 2002): urban-industrial aerosol, 
biomass burning aerosol, desert dust aerosol and oceanic aerosol. For each type, the climatology of microphysical properties is given for a number of locations (see Table 1). The microphysical properties of aerosols are defined by a complex index of refraction (with real part $n$ and imaginary part $k$ ) and size distribution which is modeled as log-normal bimodal:

$$
\frac{\mathrm{d} V(r)}{\mathrm{d} \ln r}=\sum_{i=1}^{2} \frac{C_{V, i}}{\sqrt{2 \pi} \sigma_{i}} \exp \left[-\frac{\left(\ln r-\ln r_{V, i}\right)^{2}}{2 \sigma_{i}}\right] .
$$

Parameters $C_{V, i}, r_{V, i}$ and $\sigma_{i}$ are particle volume concentration, median radius and standard deviation, respectively, for two modes, a fine and a coarse mode. We denote the ratio of fine and coarse mode concentration as fine mode fraction (FMF).

Here we only consider homogeneous, spherical particles as a simplification to apply Mie theory (Mie, 1908). This yields the scattering phase matrix, which is then used as input for the radiative transfer model. Note that for the scattering phase matrix, only the FMF is relevant, not the absolute values of the volume concentrations $C_{V, i}$. The actual aerosol concentrations are scaled implicitly by setting the AOD in MYSTIC. The aerosol vertical distribution is taken as constant up to the height of $5 \mathrm{~km}$ and is zero above this height. Table 1 summarizes the values used for the Mie calculations. We applied the well-validated Mie code by Wiscombe (Wiscombe, 1980) which is implemented in the libRadtran toolbox (Mayer and Kylling, 2005). The size distribution has been set up with $r_{\min }=0.001 \mu \mathrm{m}$ and $r_{\max }=20 \mu \mathrm{m}$ with 2000 radii with equal spacing in the logarithmic scale. The number of Legendre polynomials describing the phase functions is 1000 , which is sufficient here because sharp forward scattering peaks are not relevant, as shown later.

An example of three simulated Stokes maps of $I, Q$ and $U$ is shown in Fig. 1. The maps have been modeled for a SZA of $60^{\circ}$ at a wavelength of $650 \mathrm{~nm}$ and the urban aerosol type a (Greenbelt, USA) with an aerosol optical depth (AOD) of 0.23 . The maps are oriented such that the PP is horizontal. The center of the projections coincides with the zenith and the outer circle corresponds to the horizon. The sun's azimuth angle is $0^{\circ}$. The aureole is clearly visible in the $I$-component.

\subsection{Image analysis}

There exists one fundamental difference between the conventions of all-sky imaging and radiative transfer modeling, which has adopted the convention of the scanning method. For a sky-scanning instrument, the reference angle for the Stokes vector is defined with respect to the viewing direction, i.e. the instrument reference frame rotates along with the viewing azimuth angle. In the imaging method (using a rotating polarizer in the image plane), the Stokes reference angle is constant across the image plane, independent of the viewing azimuth angle.
The Stokes maps must therefore be transformed to allow comparison of imaging and sky scanning method or model output. The transformation from the co-rotating to fixed reference frame is realized by a linear transformation, the $2 \times 2$ rotation matrix with rotation angle $2 \varphi$, where $\varphi$ is the azimuth:

$\left(\begin{array}{c}Q_{\mathrm{r}} \\ U_{\mathrm{r}}\end{array}\right)=\left(\begin{array}{cc}\cos 2 \varphi & \sin 2 \varphi \\ -\sin 2 \varphi & \cos 2 \varphi\end{array}\right)\left(\begin{array}{c}Q \\ U\end{array}\right)$.

The first Stokes component (the radiance $I$ ) is unaffected by the transformation. $Q_{\mathrm{r}}$ and $U_{\mathrm{r}}$ are the rotated Stokes parameters that will be determined with a polarized all-sky imager, applying Eq. (1) at each pixel. In Fig. 2, the maps from the above example (in Fig. 1) are shown in the rotated basis, $Q_{\mathrm{r}}$ and $U_{\mathrm{r}}$. Note that the rotated maps are smooth over the projection, and the singularity at the zenith is eliminated by the transformation. This will be a useful advantage for the image analysis in the next section.

Finally, we normalize the rotated Stokes components $Q_{\mathrm{r}}$ and $U_{\mathrm{r}}$ with the radiance $I$ at each pixel, so we consider the relative quantities $Q_{\mathrm{r}} / I$ and $U_{\mathrm{r}} / I$. These relative Stokes maps are shown in Fig. 3. This has two distinct advantages, especially when using a commercial digital camera for imaging.

First, absolute radiometric calibration is elaborate, has to be performed regularly and typically requires temperature stabilization in field operation. Even then, the calibration accuracy is of the order of $5 \%$. Second, the aureole is generally difficult for analysis because of the large difference of direct sun irradiance and sky radiance, leading to overexposed pixels and lens flare. In the normalized Stokes maps, the aureole is weighted least and is naturally eliminated. So our processing eludes the requirement of absolute calibration and reduces the impact of undesired aureole artifacts.

The next question we address is the efficiency of data representation in the Stokes maps, to make it more feasible for a retrieval algorithm. The data considered here, involve two relative Stokes maps at three wavelengths, i.e. minimum of $25 \times 25$ pixels $\times 2 \times 3$, on the order of $10^{3}$ data points. Real data from digital images may be even higher by a factor of more than 1000 . This number is neither efficient in data representation, nor tractable for an inverse problem involving optimization.

Any function can be represented as coefficients of basis functions, such as a Fourier series. For the hemispherical projections considered here, 2-D functions, such as the Zernike polynomials are well suited. Zernike polynomials define an orthonormal basis on the unit disk and are commonly used in applied optics to describe imaging distortions (Born and Wolf, 1999). The Zernike polynomials are indexed by two integers, $n$ and $m$, associated with radial and azimuthal degrees of freedom. Here we use a single ordering number, derived by first sorting the radial, then the azimuthal number. The first orders correspond to $(n, m):(0,0),(1,-1),(1,1),(2,-2)$, $(2,0)$, etc. 
Table 1. Microphysical properties of the four representative aerosol types observed at different locations. Urban aerosol: Greenbelt, USA, (a), Paris, France (b), Mexico City, Mexico (c), Maldives (d). Biomass burning aerosol: Amazonian forest, Brazil (e), South American cerrado, Brazil (f), African Savanna, Zambia (g), Boreal forest, USA/Canada (h). Desert dust aerosol: Bahrain, Persian Gulf (i), Solar Village, Saudi Arabia (j), Cape Verde (k). Oceanic aerosol: Lanai, Hawaii, USA (l).

\begin{tabular}{|c|c|c|c|c|c|c|c|c|}
\hline & & Urban & & Biomass & & Desert & & Oceanic \\
\hline \multirow{4}{*}{$n, k$} & $\mathrm{a}$ & $1.40,0.003$ & $\mathrm{e}$ & $1.47,0.00093$ & $\mathrm{i}$ & $1.55,0.0025$ & 1 & $1.36,0.0015$ \\
\hline & $\mathrm{b}$ & $1.40,0.009$ & $\mathrm{f}$ & $1.52,0.015$ & $\mathrm{j}$ & $1.56,0.0029$ & & \\
\hline & $\mathrm{c}$ & $1.47,0.014$ & $\mathrm{~g}$ & $1.51,0.021$ & $\mathrm{k}$ & $1.48,0.0025$ & & \\
\hline & $\mathrm{d}$ & $1.44,0.011$ & $\mathrm{~h}$ & $1.50,0.0094$ & & & & \\
\hline \multirow{4}{*}{$r_{V f}, r_{V c}(\mu \mathrm{m})$} & $\mathrm{a}$ & $0.16,3.23$ & $\mathrm{e}$ & $0.15,3.50$ & $\mathrm{i}$ & $0.15,2.54$ & 1 & $0.16,2.70$ \\
\hline & $\mathrm{b}$ & $0.16,2.95$ & $\mathrm{f}$ & $0.14,3.50$ & $\mathrm{j}$ & $0.12,2.32$ & & \\
\hline & $\mathrm{c}$ & $0.14,2.96$ & $\mathrm{~g}$ & $0.13,3.50$ & $\mathrm{k}$ & $0.12,1.90$ & & \\
\hline & $\mathrm{d}$ & $0.18,2.86$ & $\mathrm{~h}$ & $0.16,3.29$ & & & & \\
\hline \multirow{4}{*}{$\sigma_{f}, \sigma_{c}$} & $\mathrm{a}$ & $0.38,0.75$ & $\mathrm{e}$ & $0.40,0.79$ & $\mathrm{i}$ & $0.42,0.61$ & 1 & $0.48,0.68$ \\
\hline & $\mathrm{b}$ & $0.43,0.79$ & $\mathrm{f}$ & $0.47,0.70$ & $\mathrm{j}$ & $0.40,0.60$ & & \\
\hline & $\mathrm{c}$ & $0.43,0.63$ & $\mathrm{~g}$ & $0.40,0.73$ & $\mathrm{k}$ & $0.53,0.59$ & & \\
\hline & $\mathrm{d}$ & $0.46,0.76$ & $\mathrm{~h}$ & $0.43,0.81$ & & & & \\
\hline \multirow{4}{*}{$C_{V f}, C_{V c}\left(\mu \mathrm{m}^{3} / \mu \mathrm{m}^{2}\right)$} & $\mathrm{a}$ & $0.060,0.026$ & $\mathrm{e}$ & $0.048,0.020$ & $\mathrm{i}$ & $0.060,0.35$ & 1 & $0.16,0.32$ \\
\hline & $\mathrm{b}$ & $0.058,0.030$ & $\mathrm{f}$ & $0.040,0.052$ & $\mathrm{j}$ & $0.028,0.37$ & & \\
\hline & $\mathrm{c}$ & $0.048,0.044$ & $\mathrm{~g}$ & $0.048,0.036$ & $\mathrm{k}$ & $0.028,0.36$ & & \\
\hline & $\mathrm{d}$ & $0.048,0.060$ & $\mathrm{~h}$ & $0.050,0.022$ & & & & \\
\hline \multirow{4}{*}{ FMF } & $\mathrm{a}$ & 2.3 & $\mathrm{e}$ & 2.4 & $\mathrm{i}$ & 0.17 & 1 & 0.50 \\
\hline & $\mathrm{b}$ & 1.9 & $\mathrm{f}$ & 0.77 & $\mathrm{j}$ & 0.075 & & \\
\hline & $\mathrm{c}$ & 1.1 & $\mathrm{~g}$ & 1.3 & $\mathrm{k}$ & 0.078 & & \\
\hline & $\mathrm{d}$ & 0.80 & $\mathrm{~h}$ & 2.3 & & & & \\
\hline
\end{tabular}
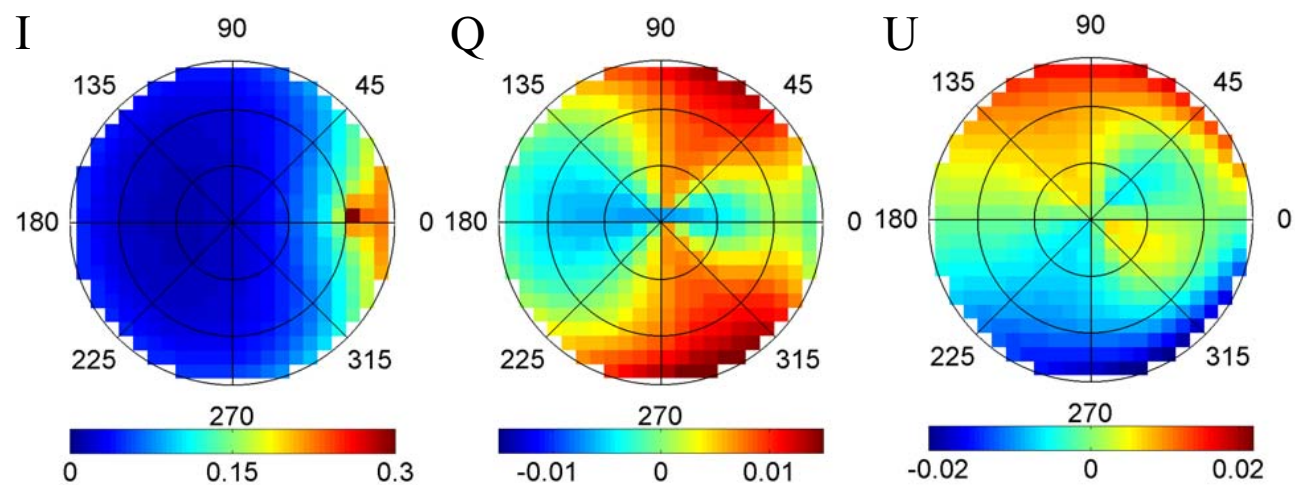

Fig. 1. Polar plots of the Stokes maps $I, Q$ and $U$ from model calculations for $650 \mathrm{~nm}, \mathrm{SZA}=60^{\circ}$ and urban aerosol type a (Greenbelt, USA) with $\mathrm{AOD}=0.23$. Azimuth angles are shown, while the zenith angles are indicated as concentric circles of 30,60 and $85^{\circ}$, respectively. The radiance values are normalized to the extraterrestrial irradiance.

By describing the relative Stokes maps $Q_{\mathrm{r}} / I$ and $U_{\mathrm{r}} / I$ in terms of the first few Zernike coefficients (ZC), we can considerably reduce the number of independent measurement parameters. The first ten $\mathrm{ZC}$ are shown in Fig. 4, together with polar plots of the first Zernike polynomials. Note that Zernike polynomials of order $1,3,5,6,9$ and 10 are symmetric about the principal plane and only the corresponding ZC of the symmetric $Q_{\mathrm{r}} / I$ maps are non-zero. The antisymmetric $U_{\mathrm{r}} / I$ maps are described exclusively by the $\mathrm{ZC}$ of the anti-symmetric polynomials of order $2,4,7$ and 8 . So in that sense, the $Q_{\mathrm{r}} / I$ maps and $U_{\mathrm{r}} / I$ maps are mutually orthogonal and the ten-component vectors of $\mathrm{ZC}$ of each relative Stokes map can simply be added, which is equivalent to the first ten $\mathrm{ZC}$ of the sum of the maps $\left(Q_{\mathrm{r}}+U_{r}\right) / I$.

Concatenating the ZCs of all three wavelengths 650, 550 and $450 \mathrm{~nm}$ yields a 30-component vector representing our data. In pattern recognition and machine vision research, such a measurement vector is commonly called feature vector (FV), a term we will also adopt in this work. 

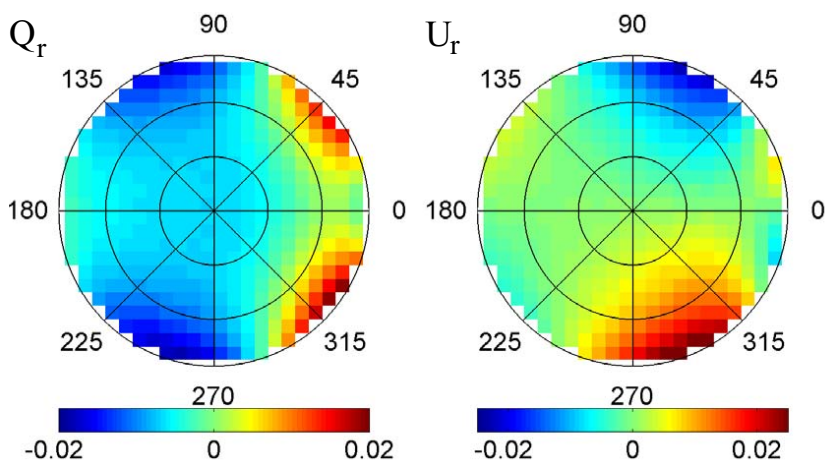

Fig. 2. Rotated Stokes maps $Q_{\mathrm{r}}$ and $U_{\mathrm{r}}$ from model calculations for $650 \mathrm{~nm}, \mathrm{SZA}=60^{\circ}$ and urban aerosol type a (Greenbelt, USA) with $\mathrm{AOD}=0.23$
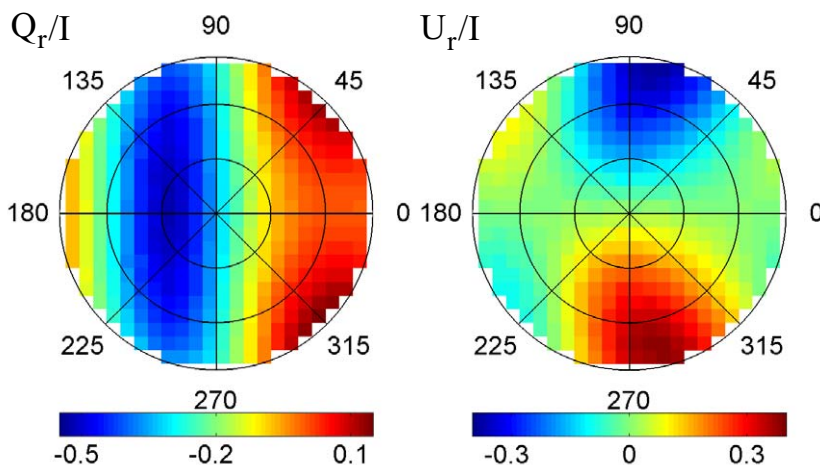

Fig. 3. Rotated relative Stokes maps $Q_{\mathrm{r}} / I$ and $U_{\mathrm{r}} / I$ from model calculations for $650 \mathrm{~nm}, \mathrm{SZA}=60^{\circ}$ and urban aerosol type a (Greenbelt, USA) with $\mathrm{AOD}=0.23$.

\section{Results}

\subsection{Aerosol FV space representation}

We simulate the Stokes maps for the 12 aerosol properties from Table 1 for $\mathrm{SZA}=60^{\circ}$ and the AOD at $450 \mathrm{~nm}$ set to 0.4 . The AODs at 550 and $650 \mathrm{~nm}$ are determined by the Ångström exponent $\alpha$ which is the negative slope of $\log (\mathrm{AOD})$ plotted against $\log (\lambda)$ (e.g. Eck et al., 1999). Here, $\alpha$ is taken as 1.5 for fine mode dominated urban and biomass burning aerosols and 0.5 for coarse mode dominated desert and oceanic aerosols, which are typical values for these types of aerosols (Dubovik et al., 2002; Eck et al., 1999). The AODs are listed in Table 2.

The resulting 12 FVs (a-1) span the FV space. We standardize the FV components so that the mean of the distribution of each component is zero. The standard deviation is not normalized to unity since the absolute and not the relative errors of the FV components are roughly constant. The standardized FV space is shown as a matrix in Fig. 5, where the rows are the FVs of each aerosol. In general, the variation of the FV components between the aerosols is increasing with wavelength because of decreasing multiple scattering

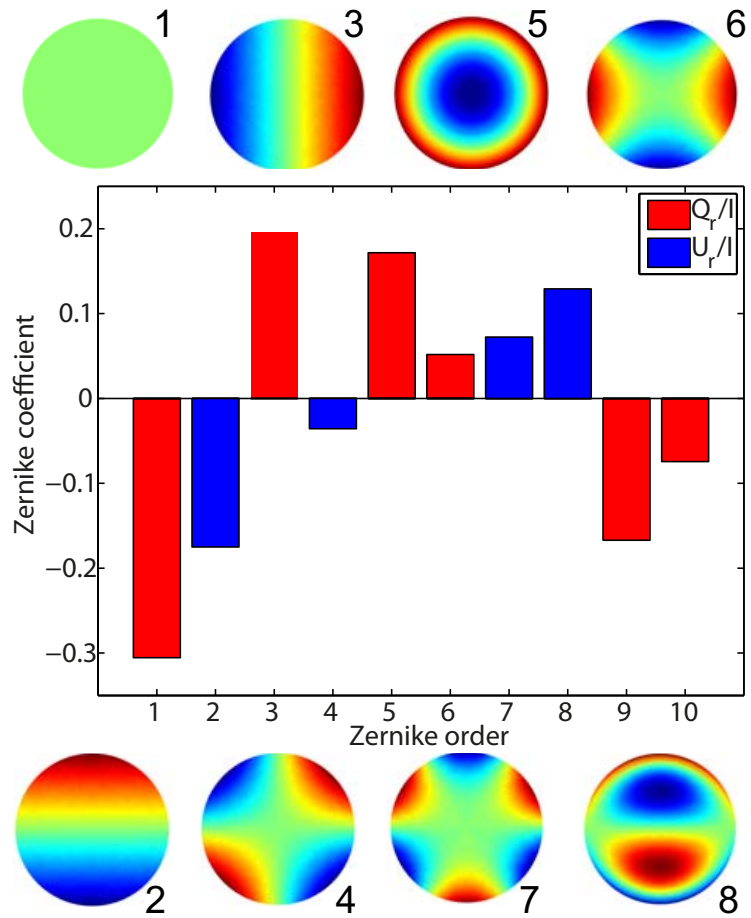

Fig. 4. The first ten Zernike coefficients for the maps of $Q_{\mathrm{r}} / I$ and $U_{\mathrm{r}} / I$, defining the feature vector for $650 \mathrm{~nm}$. Shown above are polar plots of the first four symmetric Zernike polynomials (order 1, 3, 5 and 6) yielding the coefficients for the maps of $Q_{\mathrm{r}} / I$. The antisymmetric Zernike polynomials (order 2, 4, 7 and 8) yielding the coefficients for the maps of $U_{\mathrm{r}} / I$ are shown at the bottom. The polar axes are the same as for the Stokes maps in Figs. 1-3.

Table 2. AODs used for modeling the aerosol scenarios. The AOD at $450 \mathrm{~nm}$ determines the AOD at $550 \mathrm{~nm}$ and $650 \mathrm{~nm}$ by the Ångström relation with $\alpha=1.5$ for fine mode dominated urban and biomass burning aerosol and $\alpha=0.5$ for coarse mode dominated desert and oceanic aerosol.

\begin{tabular}{lccccc}
\hline & \multicolumn{2}{c}{ AOD $_{550}$} & & \multicolumn{2}{c}{ AOD $_{650}$} \\
\cline { 2 - 3 } \cline { 5 - 6 } AOD $_{450}$ & $\begin{array}{c}\text { Urban/ } \\
\text { Biomass }\end{array}$ & $\begin{array}{c}\text { Desert/ } \\
\text { Oceanic }\end{array}$ & & $\begin{array}{c}\text { Urban/ } \\
\text { Biomass }\end{array}$ & $\begin{array}{c}\text { Desert/ } \\
\text { Oceanic }\end{array}$ \\
\hline 0.4 & 0.30 & 0.36 & & 0.23 & 0.33 \\
0.2 & 0.15 & 0.18 & & 0.12 & 0.17 \\
0.1 & 0.07 & 0.09 & & 0.06 & 0.08 \\
\hline
\end{tabular}

and a decreasing contribution from Rayleigh scattering in the Stokes maps. Note also that the desert type aerosols (i-k) stand out against the other types.

The FV space is a multivariate distribution, showing significant correlation between its components. This is because the characteristic shape of the Stokes maps is largely invariant under the influence of different aerosol types and mainly the amplitude changes, affecting all ZCs in a similar way. Considering this correlation, the number of independent 


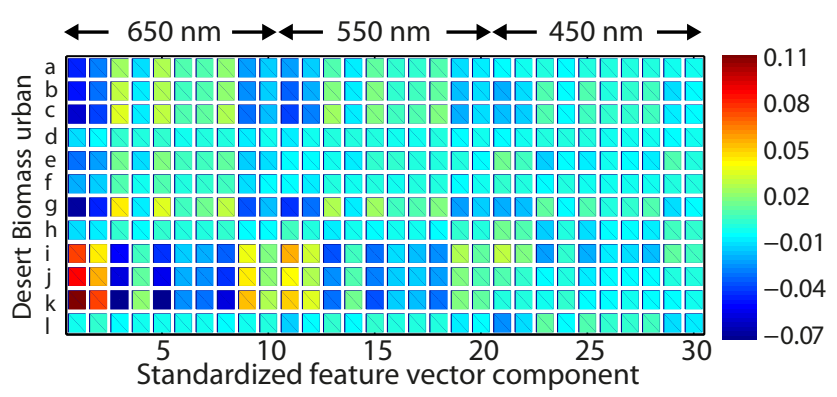

Fig. 5. Standardized feature vector space for representative aerosol components: Rows correspond to FVs for the 12 aerosols (a-l) as specified in Table 1. FV-components $1-10$ correspond to $650 \mathrm{~nm}$, components $11-20$ to $550 \mathrm{~nm}$ and $21-30$ to $450 \mathrm{~nm}$. Standardization is such that the mean of each column is zero.

parameters in the FV can be further reduced. The rotation matrix that diagonalizes the covariance matrix of the $\mathrm{FV}$ space projects each FV onto the so-called principal axes. The eigenvalues of the rotation matrix are the variances along each axis. In case of high correlation, most eigenvalues are close to zero and only the first few coordinates in this transformed coordinate system have to be considered. This is a common tool in statistical analysis, called principal component analysis (PCA).

We find that the first two PCs relate to $99 \%$ of the total variance. So the 30 -dimensional FV can be projected onto a plane in two dimensions with the highest variances, with the first two PCs being the new coordinates. In this PC representation, the FV space is conveniently visualized as shown in Fig. 6a. All aerosols are clearly distinguishable. The desert type aerosols are clustered at high PC1 values around 0.15 , while urban and biomass burning aerosols tending towards lower PC1 values of around -0.5 . Urban and biomass burning aerosols overlap partially, with biomass burning aerosols tending towards a higher PC2 than urban.

To gauge the distance between the points in Fig. 6a they have to be compared with the precision with which they can be determined from a measurement. Since the PCs are indirectly derived from the Zernike coefficients, analytical error propagation is not suitable here and we estimate the measurement error in PC space by a Monte Carlo method. We add random, Gaussian noise to the modeled radiances and simulate the complete measurement process, i.e. computing $I_{0}, I_{45}, I_{90}$ and $I_{135}$ and deriving the $I, Q$ and $U$. Then we rotate the 50 generated FVs into PC space.

A statistical error of $5 \%$ of the lowest intensity (which coincides to about $90^{\circ}$ scattering angle) has been added as random pixel noise. The resulting relative error is only around $1 \%$ for the first two FV components and in PC space corresponds to a standard deviation of $\mathrm{PC} 1$ and $\mathrm{PC} 2$ of 0.0012 and 0.0017 , respectively. The standard deviations of PC2 are plotted as error bars in Fig. 6a. Due to the larger $x$ axis scale (by a factor of five), the error bars of PC1 are negligibly small in the plot and have been omitted.

Note that there are also uncertainties associated with the microphysical properties in Table 1 due to the uncertainties in the retrieval (Dubovik et al., 2000). These may in some cases be considerable (e.g. for the values of $k$ if $k$ is small) and may also deviate from reported in situ measurements, but are not relevant for our argument and are not considered here.

\subsection{Effect of aerosol properties and SZA}

We now investigate the individual influences of the microphysical properties to see the effect on their PCs. We focus on two specific aerosols, the urban type a (Greenbelt, USA) and desert type i (Bahrain, Persian Gulf). Keeping all other parameters constant we individually vary $n, k$ and the FMF. The result of this variation is depicted in Fig. $6 \mathrm{~b}$.

Increasing $n$ of the urban aerosol from 1.4 to 1.5 causes both PCs to increases. The same effect is observed in opposite direction when $n$ of the desert aerosol is decreased from 1.55 to 1.45 and 1.4. Varying $k$ has the opposite effect as varying $n$, as noticeable for both aerosol types. A higher $n$ and lower $k$ implies enhanced scattering and lower values of $Q$ and $U$.

Then we look at the effect of a changing FMF. Decreasing the FMF of the urban aerosol from 2.3 down to 0.2, has the effect of increasing PC1 and decreasing PC2. A higher FMF than 2.3 has negligible effect, the fine mode seems to be already dominant. For the desert aerosol the original FMF is considerably lower at 0.17 , but the effect of a FMF variation is in the same direction as for the urban aerosol.

So with these observations, we better understand the distribution of the aerosols in the PC representation in Fig. 6a. The most prominent difference between the urban/biomass and the desert aerosol is their size distribution or FMF. Urban and biomass aerosols are fine-mode dominated, typically with FMF $>1$ and are separated from the coarse mode dominated desert aerosols $(\mathrm{FMF}<0.2)$ by a lower PC1. Note that in terms of volume concentrations, the coarse mode is over represented as compared to number concentrations, since large particles contribute more effectively to volume. So in terms of number concentrations, the ratio of fine and coarse mode concentrations is much larger.

Biomass aerosols are generally characterized by a higher $n$ around 1.5 as compared to a typical $n$ of 1.4 for urban aerosols. This explains the tendency to higher PC2. However, the absorption parameter $k$ of biomass aerosols may vary considerably depending on details of the combustion process. African savanna fires (aerosol type g) are typically in the flaming phase producing significant amounts of black carbon and thus exhibit a higher $k$. In PC space it almost coincides with the most absorbing urban aerosol from Mexico City (c). The aerosols from smoldering phase dominated 

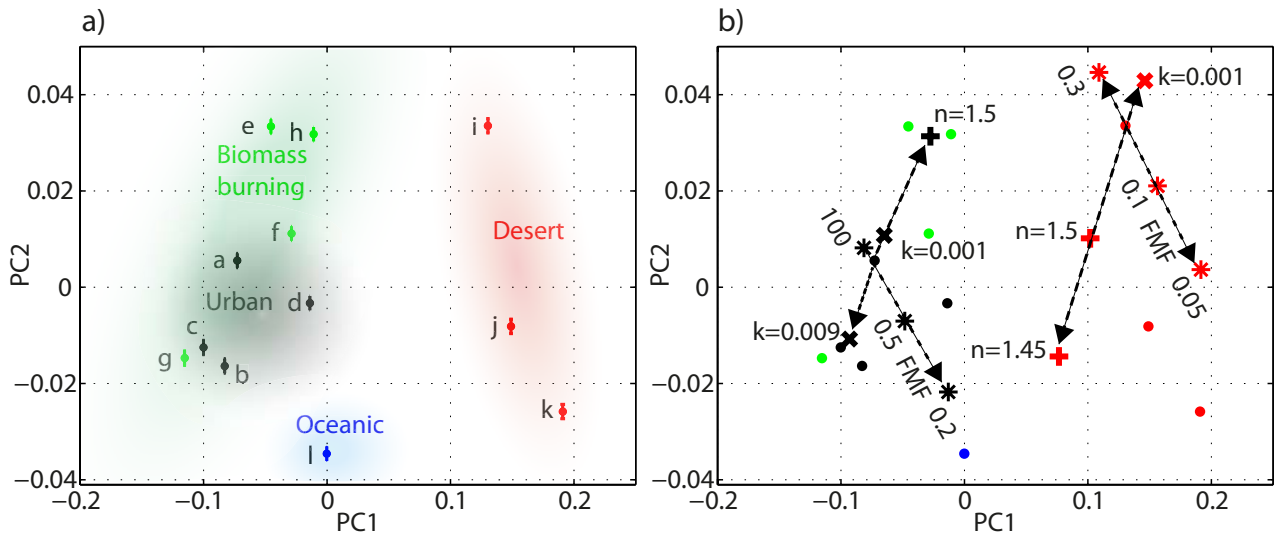

Fig. 6. (a) First two principal components of the feature vector space of the four representative aerosol types: urban (a-d), biomass burning (e-h), desert (i-k) and oceanic (1). The colored shading is meant to guide the eye in grouping the points. (b) Same as (a) but with individually varied microphysical properties of urban aerosol type a and desert aerosol type $i$, keeping all other parameters constant at the values given in Table 1. Varying $n$ is indicated with markers "+", varying $k$ with markers "x" and FMF with markers "**”.

amazon forest fires (aerosol type e) are much less absorptive and range at the high end of PC2.

The desert type aerosols are distinguishable by their small FMF and consequent high PC1. The desert aerosol from the island Cape Verde $(\mathrm{k})$ has the smallest $n$ of 1.48 , maybe from humidified particles, resulting in such a small PC2 similar to the oceanic aerosol. Oceanic aerosols from Lanai, Hawaii (1) mainly contain sea salt and water soluble particles and are characterized by the lowest $n$ of 1.36 and consequently the lowest PC2. Its FMF is 0.5 , higher than desert aerosols and lower than urban aerosols, so it occupies the intermediate PC1 range.

Next, we investigate the influence of AOD on the FV space. The AOD is determined by the total columnar aerosol concentration and may vary considerably between locations. For urban aerosols, e.g. the average measured AOD is 0.24 at $440 \mathrm{~nm}$ in Greenbelt, USA, while in Mexico City the average AOD is 0.43. The biomass burning in the South American cerrado, Brazil causes an average AOD of as high as 0.8 , while at the pristine location of Lanai, Hawaii, the AOD averages at 0.04 .

The AOD has a strong influence on the polarized radiance and for a direct comparison between aerosol types, we simulated all types with the same AOD at $450 \mathrm{~nm}$ for Fig. 6. Now, we generate the same FV space for lower AODs of 0.2 and 0.1 at $450 \mathrm{~nm}$. The corresponding AOD at 550 and $650 \mathrm{~nm}$ are again determined by the Ångström relation with $\alpha=1.5$ for urban and biomass aerosols and $\alpha=0.5$ for desert and oceanic aerosols and are given in Table 2.

The effect of decreasing AOD is shown in Fig. 7a. The PCs of the aerosol types move closer together, i.e. the FV space area is shrinking, which is related to the information content of the FV with respect to the aerosol properties. Also with decreasing AOD, the error bars decrease slightly because the radiance decreases and hence the noise in our noise model.
Of course, the less the amount of aerosols that influence the radiance, the less distinguishable the different types become. Nevertheless, even at a relatively low AOD of 0.1, the different aerosols types are still clearly distinguishable. The quality assurance criteria for the AERONET retrieval of $n$ and $k$ restrict the AOD > 0.4 at $440 \mathrm{~nm}$ (Holben et al., 2013).

Finally, we investigate the influence of SZA on the FV space and generate the same FV space as for Fig. 6 (AOD 0.4 ) but for lower SZAs of 45,30 and $15^{\circ}$ shown in Fig. $7 \mathrm{~b}$. With decreasing SZA, the FV space area decreases while the error bars are independent of SZA. This again corresponds to a decrease in information content of a measured FV and is a result of a reduction of observable scattering angles in the sky. Nevertheless, the different aerosol types are still distinguishable at low SZA of $15^{\circ}$. This relative insensitivity to SZA is due to the consideration of the complete sky hemisphere, and even with the sun in the zenith $(\mathrm{SZA}=0)$ scattering angles of up to $90^{\circ}$ from the horizon are included in the FV.

In the almucantar, the range of scattering angles decreases dramatically at lower SZA and retrieval schemes considering sky radiance measurements in the almucantar become more difficult (Dubovik et al., 2000) which imposes a limit on the observation schedule. The standard AERONET operation includes almucantar measurements only to at a minimum of $50^{\circ}$ SZA (Holben et al., 2013).

\section{Discussion}

\subsection{Uncertainties}

There are two types of uncertainties associated with the PC space. In Sect. 3, we have estimated the statistical uncertainties of the FV arising from random noise in the radiances measured on each pixel of an all-sky imager. The noise 
a)

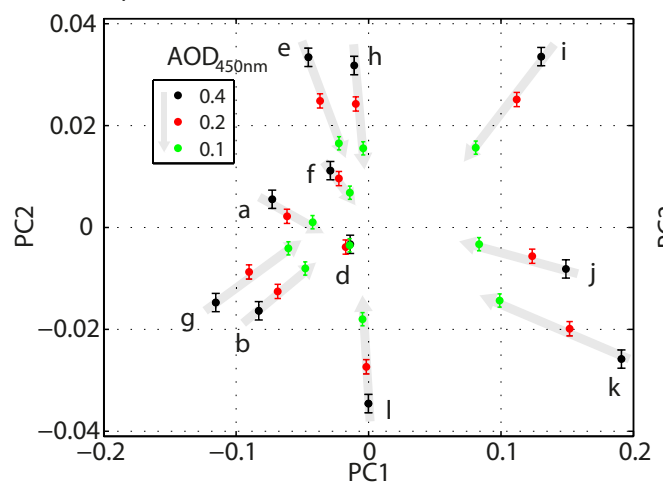

b)

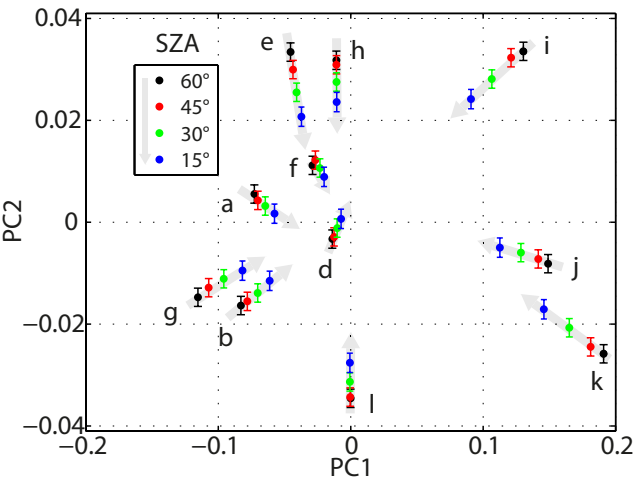

Fig. 7. (a) Effect of AOD on the PC representation of all aerosol types. The information content can be visualized as the area occupied by all points (of equal AOD) and decreases with decreasing AOD. (b) Effect of SZA on the PC representation of all aerosol types. The information content decreases with decreasing SZA.

in the radiances have been propagated to the PC-space by a Monte Carlo method (Error bars in Figs. 6a and 7a, b). The statistical errors of the FVs are indeed very small and this insensitivity to pixel noise is a result of averaging. The Zernike coefficients are a weighted sum of the whole image and any statistical noise is effectively averaged out. Real camera images typically have a lot more pixels which would further reduce the standard deviation of the PCs by an order of magnitude. However, in reality other noise sources may be more relevant such as artifacts due to dirt on the lens or obstacles on the horizon, which will have to be detected and eliminated by image processing filters using a smoothness criterion on the Stokes maps.

Another type of uncertainty is related to the modeling of aerosol scattering. It is been pointed out that desert dust aerosols are typically non-spherical and more appropriately modeled by spheroids. Ignoring this fact may cause considerable systematic errors in the retrieval of dust properties (Dubovik et al., 2006). In particular for side and backscattering angles, the scattering by spheroids is less angle dependent than scattering by spheres. But modeling the scattering of non-spherical particles is computationally far more involved than Mie calculations or requires simplified assumptions. Here we have restricted our study to spherical particles and the effect of non-sphericity on the PC representation of desert aerosols should be subject of further investigations involving, for example, the efficient T-matrix method of Mishchenko and Travis (1994).

Both types of uncertainties are relevant for the accuracy of retrieved aerosol optical properties. For quantitatively assessing the retrieval accuracy, an inversion algorithm has to be applied. In fact, the maximum likelihood estimation method that has been implemented in the AERONET scheme (Dubovik and King, 2000) could be adopted for our FV without modification. An efficient optimization procedure relies on efficient modeling of the FV and to this end, a considerable short cut has been suggested (R. Buras, personal communication, 2012). Instead of calculating the polarized radiances for each viewing angle, and hence the $\mathrm{ZC}$, the $\mathrm{ZC}$ can be calculated directly by weighting the random photon directions in the MC-model with the Zernike polynomials.

\subsection{Information content}

The information content $H$ of a measurement can be defined in terms of prior and posterior covariances of the FV space, $\mathbf{S}_{1}$ and $\mathbf{S}_{2}$ (e.g. Rodgers, 2000):

$H=\frac{1}{2} \log \left|\mathbf{S}_{1} \mathbf{S}_{2}^{-1}\right|=\frac{1}{2 s} \log \left|\mathbf{S}_{1}\right|$.

For our statistical noise model, the corresponding uncertainties in PC1 and PC2 are uncorrelated and the posterior covariance $\mathbf{S}_{2}$ can be taken as a constant $s$, shown as the error bars in Fig. 6a.

The argument of the logarithm in Eq. (4) has the dimension of a generalized volume defined by the eigenvectors of the covariance matrix. In our two-dimensional PC representation, this corresponds to the area of a rectangle with side lengths of the standard deviations of PC1 and PC 2 of the FV space.

As an easier visualization, the area occupied by all points in the FV space, can be used as a relative measure of the information content. This is a useful concept in this context to understand the effect of AOD and SZA on the FV space. We also used the idea of FV space area to find the optimal number of ZC defining the FV. The FV space area increases with increasing ZC, leveling off after ten components per wavelength. Using $16 \mathrm{ZCs}$, the area only increases by $2 \%$ compared to $10 \mathrm{ZCs}$.

Furthermore, we can compare other conceivable FV definitions with respect to their information content. For our definition, we used both relative Stokes parameters $Q_{\mathrm{r}} / I$ and $U_{\mathrm{r}} / I$ in order to involve the maximum amount of information 
accessible from polarized all-sky images. Converting the Stokes vector to spherical coordinates, it is expressed in terms of the degree and angle of polarization. The degree of linear polarization (DOLP) is the relative length of the Stokes vector: DOLP $=\frac{\sqrt{Q^{2}+U^{2}}}{I}$. We found that using the $\mathrm{ZC}$ of the DOLP maps to define the FV, the FV space in Fig. 6a remains invariant, except that the signs of $\mathrm{PC} 1$ and $\mathrm{PC} 2$ change. This may be surprising because we have reduced the number of parameters from two to one, and yet the information content is not affected. This implies that $Q$ and $U$ (or equivalently, DOLP and the angle of polarization) are correlated and do not carry independent information.

So in principle, we could also use the DOLP to define the FV, eluding the need for rotating the Stokes maps since DOLP is independent of the reference frame. Nevertheless, we keep the rotational transformation in Sect. 2.3 because it is a useful tool in the frame work of all-sky imaging and allows more flexible and general analyses regarding Stokes components.

\section{Conclusions}

We have introduced and described a 30-dimensional measurement vector (feature vector) derived from polarized allsky images. This feature vector consists of the Zernike coefficients of relative Stokes maps for three wavelengths. It ideally accommodates common challenges associated with all-sky imaging: it eludes the requirement of absolute calibration, circumvents overexposure problems of the aureole and is insensitive to pixel noise.

Using a MC radiative transfer model, we generated the FV space for realistic aerosol scenarios using the optical properties of key aerosol types retrieved from worldwide AERONET stations. Considering the covariance of the FV space, we projected the FV onto the first two principal axes with the highest variances, allowing a two dimensional representation. We have shown that, using this representation, aerosol types can be clearly distinguished with respect to size distribution and index of refraction.

Based on this work, a proper retrieval algorithm has to be implemented as a next step to quantitatively assess the accuracy of retrieved aerosol optical properties. Undoubtedly however, information of the spectral AOD, the forward scattering angles of the aureole and the absolute radiance distribution would greatly improve any retrieval method. So we see polarized all-sky imaging as a possible complimentary method to improve the existing AERONET retrievals, especially at low AOD and SZA and regarding the detection of cloud contamination.
Acknowledgements. We thank C. Emde and R. Buras for many helpful discussions regarding modeling and the use of MYSTIC.

Edited by: O. Dubovik

\section{References}

Boesche, E., Stammes, P., Ruhtz, T., Preusker, R., and Fischer J.: Effect of aerosol microphysical properties on polarization of skylight: sensitivity study and measurements, Appl. Opt., 45, 87908805, 2006.

Born, M. and Wolf, E.: Principles of Optics, 7th Ed., Cambridge, UK, Cambridge University Press, 1999.

Cazorla, A., Shields, J. E., Karr, M. E., Olmo, F. J., Burden, A., and Alados-Arboledas, L.: Technical Note: Determination of aerosol optical properties by a calibrated sky imager, Atmos. Chem. Phys., 9, 6417-6427, doi:10.5194/acp-9-6417-2009, 2009.

Dubovik, O. and King, M. D.: A flexible inversion algorithm for retrieval of aerosol optical properties from sun and sky radiance measurements, J. Geophys. Res., 105, 20673-20696, 2000.

Dubovik, O., Smirnov, A., Holben, B. N., King, M. D., Kaufman, Y. J., Eck, T. F., and Slutsker, I.: Accuracy assessment of aerosol optical properties retrieved from Aerosol Robotic Network (AERONET) Sun and Sky radiance measurements, J. Geophys. Res., 105, 9791-9806, 2000.

Dubovik, O., Holben, B. N. , Eck, T. F., Smirnov, A., Kaufman, Y. J., King, M. D., Tanré, D., and Slutsker, I.: Variability of absorption and optical properties of key aerosol types observed in worldwide locations, J. Atmos. Sci., 59, 590-608, 2002.

Dubovik, O., Sinyuk, A., Lapyonok, T., Holben, B. N., Mishchenko, M., Yang, P., Eck, T. F., Volten, H., Munoz, O., Veihelmann, B., van der Zander, W. J., Sorokin, M., and Slutsker, I.: Application of light scattering by spheroids for accounting for particle nonsphericity in remote sensing of desert dust, J. Geophys. Res., 111, D11208, doi:10.1029/2005JD006619d, 2006.

Eck, T. F., Holben, B. N., Reid, J. S., Dubovik, O., Smirnov, A., O'Neill, N. T., Slutsker, I., and Kinne, S.: Wavelength dependence of the optical depth of biomass burning, urban, and desert dust aerosols, J. Geophys. Res., 104, 31333-31349, 1999.

Emde, C., Buras, R., Mayer, B., and Blumthaler, M.: The impact of aerosols on polarized sky radiance: model development, validation, and applications, Atmos. Chem. Phys., 10, 383-396, doi:10.5194/acp-10-383-2010, 2010

Forster, P., Ramaswamy, V., Artaxo, P., et al.: Changes in Atmospheric Constituents and in Radiative Forcing, in: Climate Change 2007: The Physical Science Basis, Contribution of WG I to the Fourth AR of the IPCC, Cambridge University Press, Cambridge, United Kingdom and New York, NY, USA, 2007.

Hess, M., Koepke, P., and Schult, I.: Optical Properties of Aerosols and Clouds: The Software Package OPAC, B. Am. Meteorol Soc., 79, 831-844, 1998.

Holben, B. N., Eck, T. F., Slutsker, I., Tanre, D., Buis, J. P., Setzer, A., Vermote, E., Reagan, J. A., Kaufman, Y. J., Nakajima, T., Lavenu, F., Jankowiak, I., and Smirnov, A.: AERONET-A federated instrument network and data archive for aerosol characterization, Remote Sens. Environ., 66, 1-16, 1998.

Holben, B. N., Eck, T. F., Slutsker, I., Smirnov, A., Sinyuk, A., Schafer, J., Giles, D., and Dubovik, O.: AERONET's Version 2.0 quality assurance criteria, publicly available on the AERONET, 
available at: http://aeronet.gsfc.nasa.gov (last access: June 2013), 2013.

Kaufman, Y. J., Tanré, D., and Boucher, O.: A satellite view of aerosols in the climate system, Nature, 419, 215-223, 2002.

Kokhanovsky, A. A., Budak, V. P.,Cornet, C., Duan, M., Emde, C., Katsev, I. L., Klyukov, D. A., Korkin, S. V., Labonnote, L. C., Mayer, B., Min, Q., Nakajima, T., Ota, Y., Prikhach, A. S., Rozanov, V. V., Yokota, T., and Zege, E. P.: Benchmark results in vector atmospheric radiative transfer, J. Quant. Spectrosc. Ra., 111, 1931-1946, 2010.

Kreuter, A., Zangerl, M., Schwarzmann, M., and Blumthaler, M.: All-sky imaging: a simple and versatile system for atmospheric research, Appl. Opt., 48, 1091-1097, 2009.

Kreuter, A., Emde, C., and Blumthaler, M.: Measuring the Influence of Aerosols and Albedo on Sky Polarization, Atmos. Res., 98, 363-367, 2010.

Li, Z., Goloub, P., Dubovik, O., Blarel, L., Zhang, W., Podvin, T., Sinyuk, A., Sorokin, M., Chen, H., Holben, B., Tanre, D., Canini, M., and Buis, J.-P.: Improvements for ground-based remote sensing of atmospheric aerosol properties by additional polarimetric measurements, J. Quant. Spectrosc. Ra., 110, 1954-1961, 2009.

Liu, Y. and Voss, K.: Polarized radiance distribution measurement of skylight. II. Experiment and data, Appl. Opt., 36, 8753-8764, 1997.

Mayer, B.: Radiative transfer in the cloudy atmosphere, Euro. Phys. J. Conf., 1, 75-99, 2009.
Mayer, B. and Kylling, A.: Technical note: The libRadtran software package for radiative transfer calculations - description and examples of use, Atmos. Chem. Phys., 5, 1855-1877, doi:10.5194/acp-5-1855-2005, 2005.

Mie, G.: Beiträge zur Optik trüber Medien, speziell kolloidaler Metallösungen, Ann. Phys., 330, 377-445, 1908.

Mishchenko, M. I. and Travis, L. D.: T-matrix computations of light scattering by large spheroidal particles, Opt. Commun., 109, 16$21,1994$.

North, J. A. and Duggin, M. J.: Stokes vector imaging of the polarized sky-dome, Appl. Opt., 36, 723-730, 1997.

Pust, N. J. and Shaw, J. A.: Digital all-sky polarization imaging of partly cloudy skies, Appl. Opt. 47, H190-H198, 2008.

Rodgers, C. D.: Inverse methods for atmospheric sounding, Theory and Practice, University of Oxford, UK, 2000.

Shettle, E. P. and Fenn, R. W.: Models for the aerosols of the lower troposphere and the effects of humidity variations on their optical properties. AFGL-TR-79-0214, Air Force Cambridge Research Laboratory, Hanscom, MA, 1979.

Vermeulen, A., Devaux, C., and Herman, M.: Retrieval of the Scattering and Microphysical Properties of Aerosols from GroundBased Optical Measurements Including Polarization, I. Method, Appl. Opt., 39, 6207-6220, 2000.

Wiscombe, W.: Improved Mie scattering algorithms, Appl. Opt., 19, 1505-1509, 1980. 